\title{
Association between Job Factors, Burnout, and Preference for a New Job: a Nationally Representative Physician Survey
}

\author{
Michael T. Huber, MD', Sandra A. Ham, MS², Muneeba Qayyum, MD'3, Lana Akkari, MD³, \\ Tokunboh Olaosebikan, MD ${ }^{3}$, Joseph Abraham, MD ${ }^{3}$, and John D. Yoon, MD ${ }^{1,3}$ \\ 'Department of Medicine, University of Chicago, Chicago, IL, USA; ${ }^{2}$ Center for Health and the Social Sciences, University of Chicago, Chicago, IL, \\ USA; ${ }^{3}$ Department of Medicine, Mercy Hospital and Medical Center, Chicago, IL, USA.
}

KEY WORDS: Salary; Colleagues; Burnout; Job preference; Job satisfaction.

J Gen Intern Med 33(6):789-91

DOI: $10.1007 / \mathrm{s} 11606-018-4412-\mathrm{z}$

(c) Society of General Internal Medicine 2018

\section{INTRODUCTION}

Physician turnover contributes to decreased physician productivity, decreased quality of patient care, and increased costs to healthcare systems. ${ }^{1-5}$ We used an experimental vignette to test associations between physician demographics, salary, and working with exemplary colleagues on the likelihood of preferring a new job among burned out and non-burned out physicians in various specialties.

\section{METHODS}

A nationally representative sample of 2000 US physicians in various specialties were surveyed in 2011 . We experimentally manipulated the effect of characteristics of a hypothetical new job on physicians' willingness to change jobs from their current job to a new job: "Imagine a physician job that is similar to the position you currently have, except that in the new job you would: 1) spend ten \{fewer or more\} hours each week caring for patients, 2) care for patients who are, on average, \{healthier or less healthy\} than your current patients, and 3) earn $20 \%$ \{more or less\} in salary. The new job would not require relocation. In it you would work with colleagues who you regard as \{exemplary or mediocre\} physicians. Please indicate to what extent you would prefer your current job or this new job?" The responses were dichotomized as "prefer new job" versus "prefer current job" for analysis. Each physician was randomly allocated to one of the 16 vignette conditions. The survey also included a validated short form of the Maslach Burnout Inventory (MBI) to assess physician burnout using two items on a 7-point Likert scale. ${ }^{5}$ Burnout was defined as either High Emotional Exhaustion or High Depersonalization or Both. The sample was evaluated with Chi-square and Mann-Whitney tests. Multivariate logistic regression model was used to test the interaction between burnout, change in salary, and the presence of mediocre versus exemplary colleagues, adjusted for work hours and patient acuity. Further details on our methodology were described in a previous study. ${ }^{6}$

\section{RESULTS}

The survey response rate was 64\% (1275/2000). Across all experimental vignettes, 265 physicians $(20.8 \%)$ preferred the new job. There were no significant differences in demographics, medical specialty, or salary among physicians preferring a new job versus those preferring a current job. Three hundred ninety-one (30.7\%) of physician respondents reported burnout. Physicians reporting burnout were more likely to prefer a new job than non-burned out physicians if the new job had a higher salary and/or exemplary colleagues (Fig. 1). Physicians were less likely to prefer the new job with both lower salary and mediocre colleagues. In adjusted analyses, burned out physicians had $19 \%$ higher odds of choosing a job with higher salary and exemplary colleagues. Burned out physicians were also over threefold more likely than nonburned out physicians to prefer the new job if salary was higher but colleagues were mediocre $(\mathrm{OR}=3.13,95 \% \mathrm{CI}=$ 3.05-3.21). In the scenario of a new job with a lower salary, burned out physicians were $81 \%$ more likely than non-burned out physicians to prefer the new job if it had exemplary colleagues but $42 \%$ less likely to prefer the new job if it had mediocre colleagues.

\section{DISCUSSION}

We found that even among physicians experiencing burnout, less than half would leave their current job for a new job with higher salary and exemplary colleagues. Among burned out physicians, the opportunity for better salary and colleagues made them more likely to prefer a new job compared to non-burned out physicians, with change in salary associated with the greatest difference. Burned out physicians were even willing to 


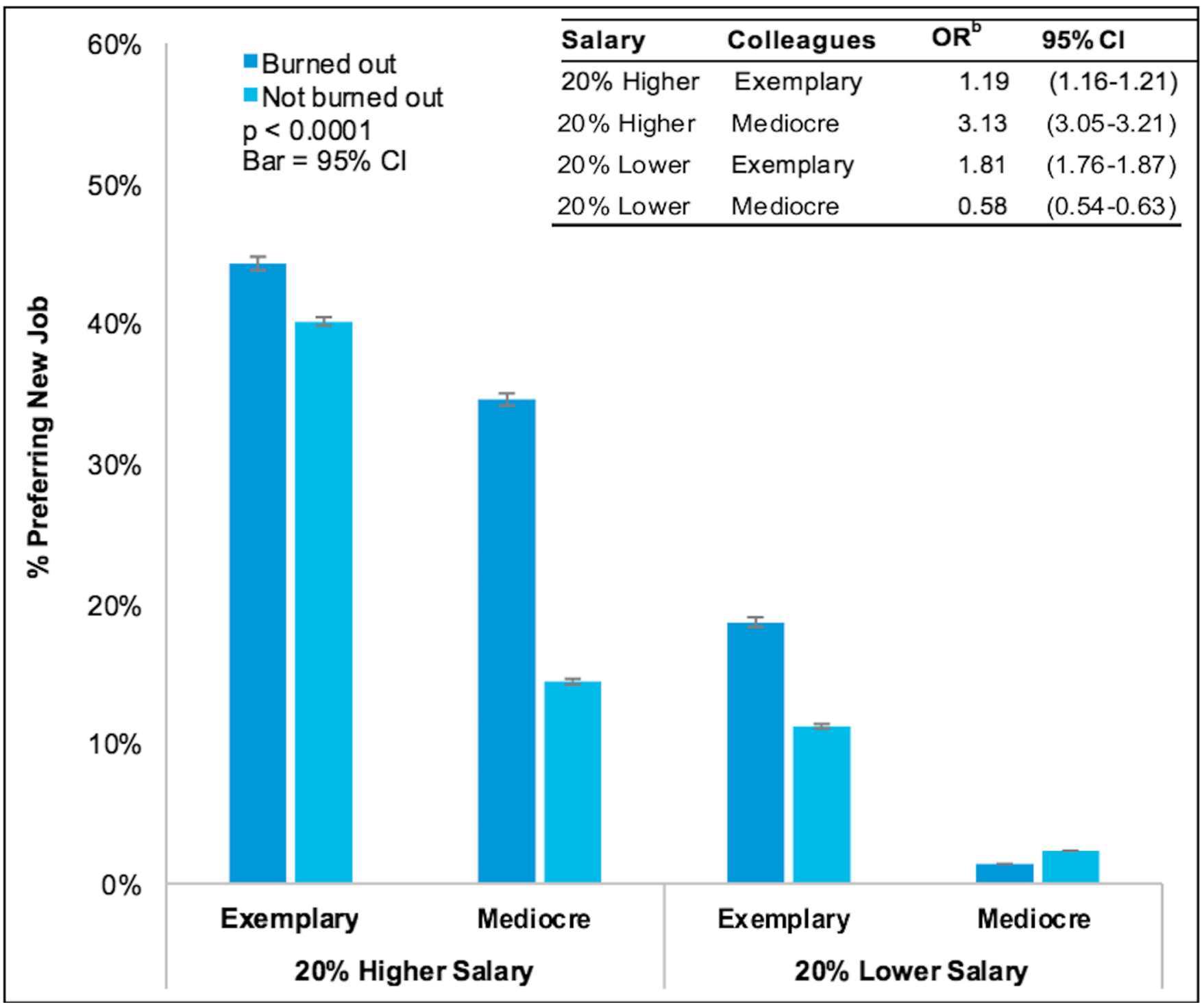

Figure 1 Preference for the new job for vignettes that varied by salary and type of colleagues, by burnout status of physician, Job Satisfaction and Meaning in the Practice of Medicine: A National Physician Study $(N=1275)$. ${ }^{2}$ Adjusted for vignette conditions hours of patient contact per week and patient health, weighted to the national sample of physicians. ${ }^{b} \mathrm{OR}=$ for each vignette condition, odds of preference for new job by burned out physicians/odds of preference for new job by physicians who were not burned out.

accept a new job with a lower salary if it had exemplary colleagues, suggesting a possible lifeline for burned out physicians who are considering "greener grass" outside their current practices.

Limitations of the study include the possibility of nonresponse bias, the cross-sectional design of old data (2011) which limit inferences on causation, our inability to explore the impact of uncertainty about whether potential colleagues were exemplary or mediocre and that our analyses did not adjust for other personal and work environment factors relevant to burnout.

Despite these limitations, this study suggests that efforts to reduce turnover must account for complex interactions affecting physician job decisions, especially in relation to intrinsic motivating factors ${ }^{6}$ that impact how physicians derive meaning and resilience from collegial relationships at work. Focusing on improving physician-colleague relationships, particularly for those experiencing burnout, may improve job retention.

Acknowledgements: We would like to thank Farr Curlin, MD for his mentorship and support of this project.

Corresponding Author: John D. Yoon, MD; Department of Medicine University of Chicago, 5841 S Maryland Ave, MC 5000, Chicago, IL 60637, USA (e-mail:jdyoon@uchicago.edu).

Source of funding Michael Huber was supported in part by a grant from the Agency for Healthcare Research and Quality (T32 HSO0O078). This study was supported by a grant from the John Templeton Foundation (Grant ID\# 13534) and by a pilot grant from the 
Bucksbaum Institute for Clinical Excellence at the University of Chicago.

\section{Compliance with ethical standards:}

Conflicts of interest: The authors declare that they have no conflicts of interest.

\section{REFERENCES}

1. Misra-Hebert AD, Kay R, Stoller JK. A review of physician turnover: rates, causes, and consequences. Am J Med Qual. 2004;19:56-66.
2. Reddy A, Pollack CE, Asch DA, Canamucio A, Werner RM. The Effect of Primary Care Provider Turnover on Patient Experience of Care and Ambulatory Quality of Care. JAMA Intern Med. 2015;356:1157-62.

3. Waldman J. Kelly D, Aurora F, Smith S, Howard L. The Shocking Cost of Turnover in Health Care. Health Care Manag Rev. 2004;29(1):2-7.

4. Linzer M., Poplau S., Grossman E. et al. A Cluster Randomized Trial of Interventions to Improve Work Conditions and Clinician Burnout in Primary Care: Results from the Healthy Work Place (HWP) Study. J Gen Intern Med. 2015;30: 1105.

5. West CP, Dyrbye LN, Sloan JA, Shanafelt TD. Single item measures of emotional exhaustion and depersonalization are useful for assessing burnout in medical professionals. J Gen Intern Med. 2009;24:1318-21.

6. Tak HJ, Curlin FA, Yoon JD. Association of intrinsic motivating factors and markers of physician well-being: A National Physician Survey. J Gen Intern Med. 2017;32(7):749-746. 